\title{
Technical Challenges in the Manufacture of Dendritic Cell Cancer Therapies
}

\author{
Miquel Lozano, Joan Cid, Daniel Benitez-Ribas, Manel Juan Otero \\ Hospital Clínic de Barcelona, Barcelona, Spain
}

DOI: https://doi.org/10.17925/EOH.2019.15.1.22

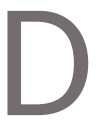
endritic cells (DCs) are the most potent antigen-presenting cells, owing to their ability to stimulate antigen-specific T cell responses. Their use as therapeutic cancer vaccines is, therefore, increasing rapidly. In order to manufacture DC vaccines, peripheral blood mononuclear cells (MNCs) are harvested by leukapheresis and enriched for monocytes by adherence to plastic, elutriation and/or CD14* selection. Differentiation of monocytes into immature DCs is then achieved by culture with cytokines. Critical issues for successful vaccination involve optimisation of cell collection, choice of antigen, antigen loading, route and schedule of administration. Regarding cell collection, apheresis systems are able to selectively collect MNCs, including monocytes, lymphocytes, CD34+ and DCs with low red blood cell, granulocyte and platelet content. This review describes the technical challenges of, and optimal procedures for, harvesting, enrichment, maturation, antigen loading and administration of DCs. Additionally, the requirement to further advance these procedures to develop this promising treatment, with the aim of achieving widespread clinical adoption of therapeutic DC-based cancer vaccines is discussed.

\section{Keywords}

Cancer vaccine manufacture, dendritic cell, leukapheresis, technical challenges of therapeutic vaccination

Disclosure: Miquel Lozano, Joan Cid, Daniel BenitezRibas and Manuel Juan Otero have no conflicts of interest to disclose relating to this manuscript.

Acknowledgements: Editorial assistance was provided by Stuart Wakelin of Touch Medical Media and funded by Terumo BCT.

Review Process: Double-blind peer review.

Compliance with Ethics: This study involves a review of the literature and did not involve any studies with human or animal subjects performed by any of the authors.

Authorship: The named authors meet the International Committee of Medical Journal Editors (ICMJE) criteria

for authorship of this manuscript, take responsibility for the integrity of the work as a whole, and have given final approval for the version to be published.

Received: 9 May 2019

Accepted: 8 July 2019

Citation: European Oncology and Haematology. 2019;15(1):22-8

Corresponding Author: Miquel Lozano, Hospital Clínic de Barcelona, Carrer de Villarroel, 170, 08036 Barcelona, Spain. E: MLOZANO@clinic.cat

Support: The publication of this article was supported by Terumo BCT, who were given the opportunity to review the article for scientific accuracy before submission. Any resulting changes were made at the authors' discretion. The views and opinions expressed are those of the authors.
Therapeutic cancer vaccination is a promising strategy that aims to treat late stage disease by using the patient's own immune system. ${ }^{1}$ The immune system has the potential to eliminate cancer cells, since cancer cells express antigens that are unique to the tumour or are over-expressed in the tumour. However, despite many years of research, the effective induction of strong and durable antitumour T-cell responses in vaccinated patients remains a challenge.' The efficient presentation of cancer antigens to T cells is key to successful vaccination; mouse models have indicated that the generation of protective antitumour immunity depends on the presentation of tumour antigens by dendritic cells (DCS). ${ }^{2}$

DCs are leukocytes derived from bone marrow and are an important component of the adaptive and innate immune system (Figure 1). They induce an immune response by presenting captured antigens to naïve T cells in lymphoid tissues. ${ }^{3}$ In addition to their immune stimulatory functions, DCs infiltrate most human tumours, where they have regulatory effects including both tumour growth inhibition and stimulation of the immunosuppressive tumour microenvironment. ${ }^{2}$ As a result, DCs have been investigated as potential therapeutic tools in cancer. ${ }^{4-12}$

DCs can be derived from monocytes or CD34+ stem cells, for example by direct isolation of peripheral blood mononuclear cells (MNCS). ${ }^{13,14}$ Autologous DCs can be generated ex vivo, activated, loaded with tumour antigens and then returned via injection to the patient with the aim of eliciting tumour-specific effector T cells that kill tumour cells and induce immunologic memory to control tumour relapse (DC vaccination). ${ }^{15,16}$ The first DC vaccination study was published in 1996 and used DCs generated ex vivo from peripheral blood monocytes to immunise B-cell lymphoma patients. ${ }^{17}$ Since then, numerous small clinical trials have evaluated DCs as cellular mediators for therapeutic vaccination of patients with cancer, many demonstrating a survival benefit. ${ }^{4-12}$ These studies have included a number of different clinical indications including: prostate cancer, ${ }^{18}$ colorectal cancer, ${ }^{19}$ melanoma, ${ }^{12,20-23}$ ovarian cancer, ${ }^{7,24}$ glioblastoma, ${ }^{8,11,25-27}$ renal cell carcinoma, ${ }^{9}$ pancreatic cancer ${ }^{28}$ and haematological malignancies. ${ }^{10}$ In 2010, the US Food and Drug Administration (FDA) licensed Sipuleucel-T for clinical use. This was the first DC-based vaccine to be approved having shown prolonged overall survival among men with metastatic castration-resistant prostate cancer29,30 and this technology has attracted considerable interest as a therapeutic option in multiple cancer types. However, while many vaccines have elicited tumour-specific immunity, objective clinical response rates remain low.

Improved techniques for harvesting DCs have resulted in many new and novel vaccine strategies being developed. This review article aims to explore the characteristics of DCs that make them an ideal choice for anti-tumour vaccines, as well as providing a detailed description of the processes and discussing the technical challenges of DC vaccine production. 
Figure 1: Role of dendritic cells in the immune response to cancer cells

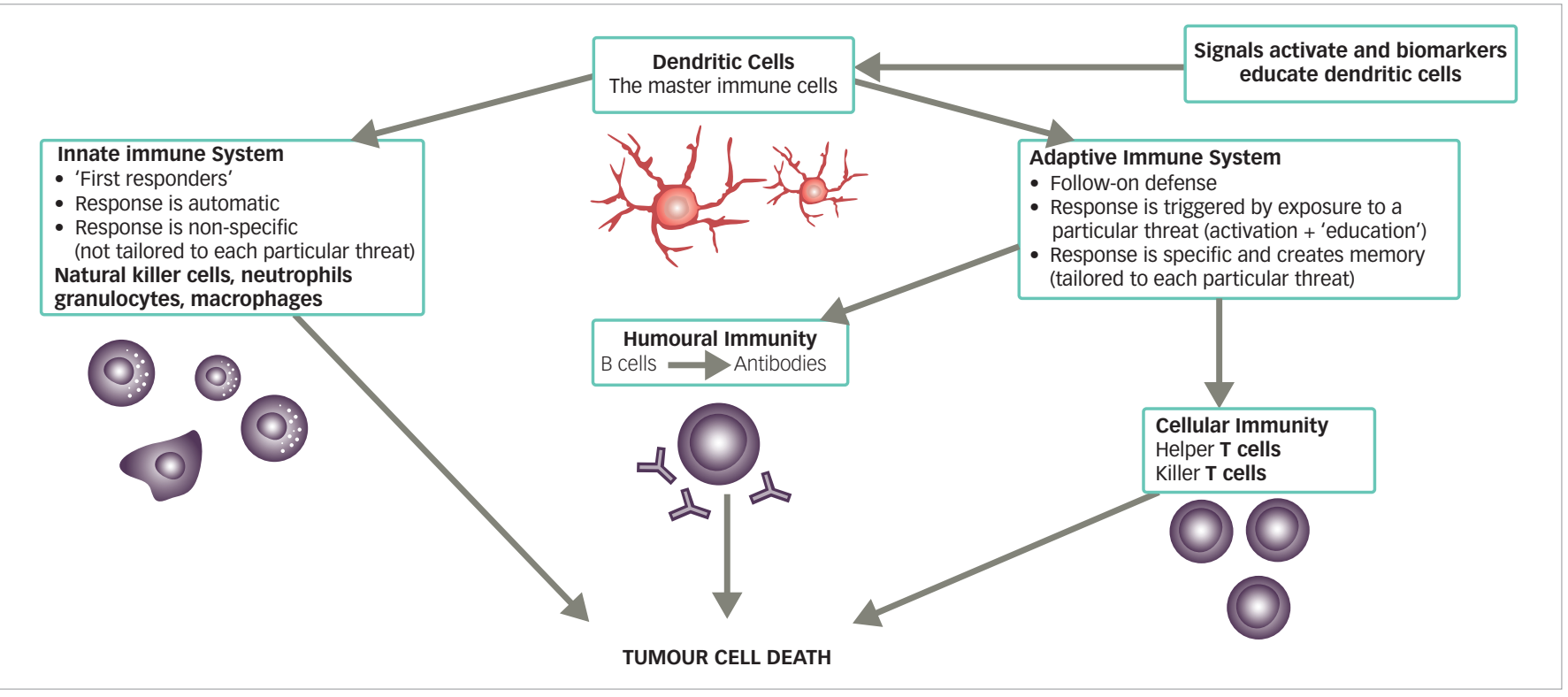

\section{Characteristics of dendritic cells}

DCs are uniquely suited to cancer immunotherapy as they form a network of antigen presenting cells (APCs) that induce primary immune responses, improve the effector functions of previously primed T-lymphocytes and facilitate cross-communication between innate and adaptive immunity (Figure 1). ${ }^{31}$ DCs are extremely efficient APCs and responses can be achieved from low numbers of $T$ cells since their potency for inducing T cell proliferation is 10-100 times that of B-cells or monocytes. ${ }^{31,32}$ They sensitise antigen-specific responses in both $\mathrm{CD}_{4}{ }^{+}$and $\mathrm{CD} 8^{+} \mathrm{T}$ cells. The latter differentiate into cytotoxic T-lymphocytes (CTLS), conferring direct anti-tumour effects. ${ }^{31}$

The other feature of DCs that makes them ideally suited for clinical use is the fact that they can be differentiated in vitro from $\mathrm{CD}_{3} 4^{+}$progenitors from purified cord blood, bone marrow or cytokine-mobilised peripheral blood progenitor cells, ${ }^{33}$ or isolated in low numbers in peripheral blood, which can be enhanced following pre-treatment with a DC-stimulating factor such as Flt3 ligand. ${ }^{16,34}$ The largest numbers of DCs can be obtained with the use of specific cytokines to mobilise CD34+ ${ }^{+}$cells from the bone marrow to the bloodstream. ${ }^{35}$ Substantial savings in materials and other costs can be achieved if monocyte-derived DC for multiple treatments are generated from cryopreserved monocytes rather than from fresh monocytes. ${ }^{36}$

\section{Generation of dendritic cell-based vaccines}

As a result of the immunosuppressive nature of most tumours, T cells activated by vaccination may become inactivated at the tumour site. High DC numbers and multiple doses of vaccine are therefore needed to assure that sufficient activated CTLS are generated to eliminate tumour cells and ensure that sufficient $T$ cells enter the memory compartment to act as rapid responders in case of tumour relapse. A typical DC vaccination dosage is $5-50$ million DC cells, and up to 15 vaccinations may be needed. ${ }^{12,37}$ The number of cells needed for immunotherapy necessitates their standardised production on a scale large enough to sustain repeated vaccinations while conforming to current good manufacturing practice (GMP) guidelines. Therefore, the generation of DC vaccines involves a number of key stages (Figure 2). Here, we elaborate on the different stages that are key for a successful DC vaccination.

\section{Harvesting}

Numerous techniques have been used to isolate DC precursors; these include apheresis, elutriation, filtration, adherence, positive selection of $\mathrm{CD} 14^{+}$monocytes and negative selection via lymphocyte depletion. ${ }^{13}$ However, harvesting by leukapheresis can generate a large number of DCs and has become the standard procedure for collection in many DCbased clinical studies. ${ }^{13}$

A number of apheresis systems are available for the harvesting of DCs. In 2003, a study compared MNC programmes on two apheresis systems (COBE ${ }^{\circledR}$ Spectra [Terumo BCT, Lakewood, Colorado, USA] and AS.TEC [Fresenius Kabi, Bad Homburg, Germany]). ${ }^{38}$ The study found that the AS.TEC cell separator showed significantly better monocyte collection rates and efficiency compared with the COBE Spectra system in $5 \mathrm{~L}$ collections (medians of $11.0 \%$ versus $7.4 \%$ [rate] and $51.9 \%$ versus $31.9 \%$ [efficiency], respectively; all $p<0.05$ ), but not in $10 \mathrm{~L}$ collections (10.4\% versus $8.5 \%$ [rate] and $67.6 \%$ versus $55.2 \%$ [efficiency], respectively; all $\mathrm{p} \geq 0.21$ ). ${ }^{38} \mathrm{In}$ both 5 and $10 \mathrm{~L}$ collections AS.TEC resulted in significantly higher residual red blood cell (RBC) yields compared with COBE Spectra (5 L: 227 versus $84 \times 10^{\circ} / \mathrm{bag}$, respectively; $10 \mathrm{~L}$ : 478 versus $142 \times 10^{\%} / \mathrm{bag}$; all $p<0.001$ ). ${ }^{38}$ To optimise the procedures, different mononuclear programme settings for COBE Spectra and AS.TEC were subsequently evaluated. For all programme settings the mean MNC purity was $\geq 93 \% .{ }^{39}$

However, use of the standard MNC programme of both devices resulted in significantly higher collection efficiencies of CD14+ monocytes, $\mathrm{CD}^{+}$ cells, CD4+ cells, CD8+ T cells, CD16+ $\mathrm{CD}^{+} 6^{+}$natural killer (NK) cells, and residual platelets (all $\mathrm{P}<0.01$ ) compared to the modified programme settings, due to a higher centrifuge speed. ${ }^{39}$ A 2005 study also compared two different settings of the COBE Spectra apheresis system: the MNC and AutoPBSC settings. ${ }^{40}$ Both settings produced similar mean yields of leucocytes (4.24 and $4.52 \times 10 \%$ unit, respectively) and lymphocytes (T cells: 2.05 and $2.16 \times 10^{\%} /$ unit). ${ }^{40}$ However, products derived using the AutoPBSC setting contained significantly $(p<0.05)$ greater mean yields of granulocytes ( 0.11 versus $0.20 \times 10^{\%} /$ unit), monocytes ( 0.98 versus $1.13 \times 10^{\%}$ /unit) and RBCs (50.9 versus $63.5 \times 10^{\%}$ /unit), but significantly fewer platelets (mean yield: 215 versus $118 \times 10^{\%} /$ unit) $^{40}$ 


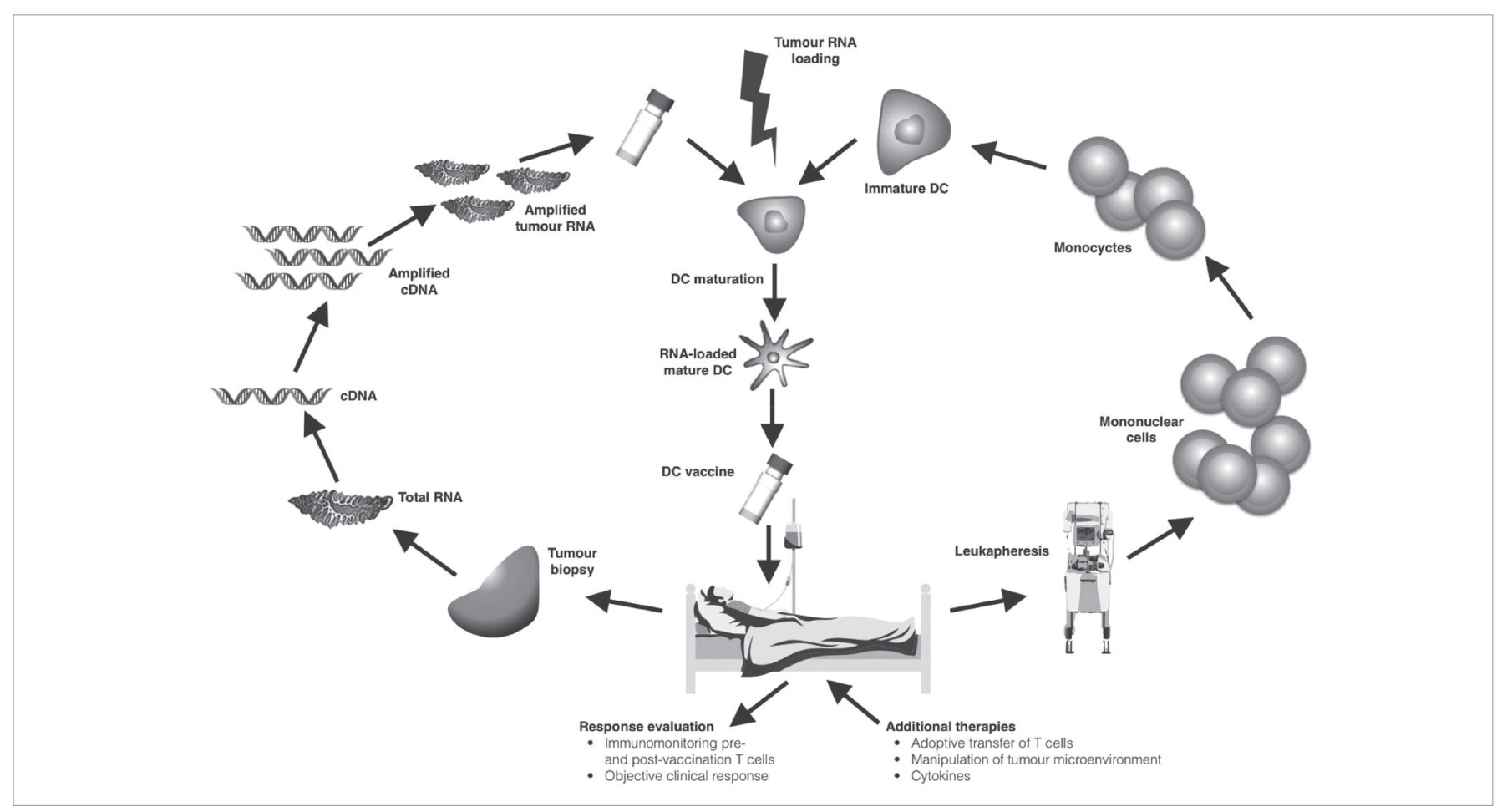

$C D N A=$ complementary $D N A ; D C=$ dendritic cell; $R N A=$ ribonucleic acid.

Different programme settings have also been evaluated for the COM.TEC ${ }^{\circledR}$ cell separator (Fresenius Kabi, Runcorn, UK). ${ }^{41}$ In 2006, twenty-four 5 L MNC collections were performed from non-cytokine-stimulated donors. The MNC setting resulted in a high mean CD14+ collection efficiency (84\%) and mean cell yield ( $1.2 \times 10 \%$ unit), but high residual platelets (mean efficiency: $42.7 \%$; mean yield: $317 \times 10 \%$ /unit). The use of a dual-stage chamber in the PBSC-LYM setting produced fewer residual platelets (mean efficiency: 9.8\%; mean yield: $79 \times 10 \%$ unit), but did not achieve the target mean $\mathrm{CD}_{14}{ }^{+}$yield of $1 \times 10^{9}$ cells. ${ }^{41}$

More recently, the Spectra Optia ${ }^{\circledR}$ apheresis system was developed (Terumo BCT). In a 2013 study, 12 MNC collections were performed using this system in non-cytokine-stimulated donors. ${ }^{42}$ This resulted in satisfactory MNC yields (including a median CD14+ of $1.28 \times 10^{\%}$ unit) with low residual RBC and platelet yields (mean: 17.0 and $104 \times 10^{\%} /$ unit, respectively). ${ }^{42}$ A 2014 study also showed that this system was able to isolate and enrich a highly purified MNC fraction (88.6\% monocytes). ${ }^{43}$ In a head-to-head study comparing Spectra Optia with other apheresis systems (COM.TEC and Amicus ${ }^{\mathrm{TM}}$ [Fresenius Kabi]), all devices resulted in similar total MNC yields. ${ }^{44}$ The Spectra Optia device, however, collected significantly more CD14+ monocytes than the Amicus device (mean yield: 1.64 versus $1.20 \times 10^{\%} ; \mathrm{p}=0.002$ ) and significantly fewer $\mathrm{CD} 3^{+}$cells (not required for DC generation) than the COM.TEC device (mean yield: 2.36 versus $3.03 \times 10^{9} ; \mathrm{p}=0.002$ ). The Spectra Optia products had the lowest mean RBC content (yield: 16.8 versus 53.0 [Amicus] and 124 [COM.TEC] $x 1^{9}$; all $p \leq 0.002$ ) while the Amicus products had the lowest mean platelet content (yield: 46.2 versus 114 [Spectra Optia] and 614 [COM. TEC] $\times 10^{9}$; all $\left.p<0.001\right) .4$

Further development of the Spectra Optia system to perform continuous MNC collection, enabling shorter procedure times with reduced product volume, showed similar (all $p \geq 0.05$ ) mean yields of monocytes
(2.91 versus $3.52 \times 10^{9}$ ) and lymphocytes (11.7 versus $12.3 \times 10^{\circ}$ ), as well as contaminating platelets, RBCs and granulocytes compared with the standard Spectra Optia MNC system..$^{45}$

Regarding the collection of other cells, a recent retrospective study compared the Spectra Optia MNC and COBE Spectra MNC systems for autologous PBSC collection and found no significant differences in the median $\mathrm{CD} 34^{+}$cell content of collected products $\left(0.61 \times 10^{8}\right.$ versus $\left.0.94 \times 10^{8}\right), C D 34+$ cell collection efficiency ( $43.5 \%$ versus $42.1 \%$ ), and loss of platelets (40.1\% versus $44.7 \%) .{ }^{46}$

Overall, modern apheresis systems have shown to be broadly similar in terms of performance and efficacy (MNC/monocyte yields and collection efficiencies). ${ }^{38,44}$ However, the Spectra Optia system has shown some benefits over other systems with regard to the collection of $\mathrm{CD} 14^{+}$ cells required for $\mathrm{DC}$ generation, and minimising $\mathrm{RBC}$ and platelet contaminants. ${ }^{44}$ Platelet contamination is a problem when monocytes are purified by adherence due to space competition as they bind to the monocytes, ${ }^{47}$ forming clumps and reducing monocyte yield..$^{38}$ To address this, reduction of the preset buffy coat volume has been shown to optimise leukapheresis harvests using the COBE Spectra system and AutoMNC setting. ${ }^{48}$ Similarly, reducing the packing factor (from 4.5 to 4.0 ) used in the Spectra Optia continuous MNC device has been shown to further reduce platelet contamination (mean total product 315 versus $494 \times 10^{9}$, respectively; $p<0.001$ ) while retaining the mean monocyte yield $\left(2.67\right.$ versus $\left.2.69 \times 10^{9} ; p \geq 0.05\right) .{ }^{45}$

\section{Enrichment}

A number of different approaches have been used for monocyte enrichment. Methods of enrichment include plastic adherence, positive selection using magnetic beads coupled to CD14 antibodies or cell depletion using beads coupled to antibodies against $C D 2^{+} T$ cells and 
Table 1: Overview of procedural characteristics for the isolation of dendritic-cell vaccine constituents using the Spectra Optia apheresis system

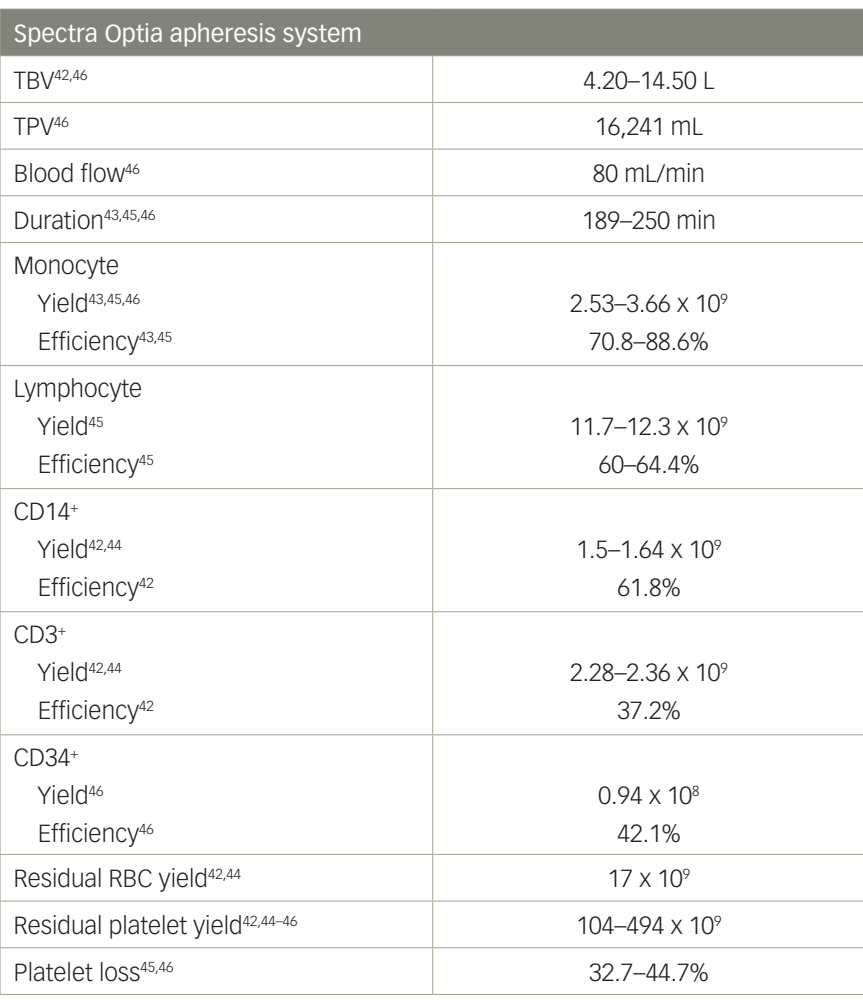

All yields in cells/unit, unless otherwise stated; ranges include standard Spectra optia MNC, continuous MNC collection, and reduced packing factor (4.0-4.5) protocols.

$M N C=$ mononuclear cell; $R B C=$ red blood cells; $T B V=$ total blood volume; TPV $=$ total product volume.

The Spectra Optia ${ }^{\circledast}$ is a product of Terumo BCT (Lakewood, Colorado, USA).

CD19+ B cells to remove non-monocytes. Available systems for these procedures include the ClinimACS $^{\circledR}$ cell-selection system (positive selection; Miltenyi, Bergisch Gladbach, Germany) and Isolex 300i Magnetic cell selector (immunomagnetic cell depletion; Nexell, Irvine, California, USA). In general, positive selection can provide a high purity of immature DCs with a low yield (e.g. $97 \pm 1 \%$ and $8 \pm 3 \%$, respectively; CliniMACS), ${ }^{49}$ whereas removal of non-monocytes provides a low purity, but a high yield $(42 \pm 10 \%$ and $21 \pm 6 \%$, respectively; Isolex $300 i){ }^{49}$ Conventional plastic adherence procedures tend to provide more balanced purity and yield $(72 \pm 4 \% \text { and } 25 \pm 5 \%)^{49}$

A study by Wong et al. also showed that it is possible to enrich monocytes by counterflow centrifugal elutriation (Beckman J-6M centrifuge; Beckman Instruments, Palo Alto, California, USA) with a similar DC purity and yield obtained using the Isolex 300i system (67-80\% and $11-13 \%$, respectively). ${ }^{50}$ The ElutraTM system (Terumo BCT) is a closed (thereby minimising the risk of contamination) counterflow centrifugal elutriation cell separation system that can also isolate monocytes from other blood components for DC generation. Monocytes are collected from the elutriation chamber in the last fraction, which also contains granulocytes, and in general the purity of the monocytes isolated using the Elutra system is at least $80 \% .^{51,52}$ The presence of up to $16 \%$ granulocytes in the monocyte product have been found to have no significant effects on the quality of the DCs formed. However, once the concentration of granulocytes increases above $16 \%$, a gradually increasing detrimental effect on DC quality is observed in terms of the migratory capacity of DCs and lower expression levels of CD80, CD40 and CD86. ${ }^{51}$ Nevertheless, the presence of $20-30 \%$ granulocytes in a monocyte product has been shown to have no major influence on the quality of the DCs generated from monocytes..$^{51}$ In conclusion, as the Elutra system isolates monocytes with a purity of $\geq 80 \%$ and can isolate up to $7 \times 10^{9} \mathrm{MNCS}^{52}$ it is a suitable system for the large-scale isolation of monocytes for the generation of DCs.

\section{Differentiation and maturation}

Differentiation of monocytes into immature DCs typically involves culture with granulocyte-macrophage colony-stimulating factor (GM-CSF) and interleukin (IL)-4 or -13, which lead to the formation of immature DCs. ${ }^{53}$ Maturation of cells is then required since mature DCs migrate more effectively than immature cells and induce better anti-tumour responses. ${ }^{54,55} \mathrm{~A}$ maturation cytokine cocktail containing IL-1beta, tumour necrosis factor (TNF)-alpha, IL-6 and prostaglandin E2 (PGE2) has been shown to yield mature DCs with high migratory potential. ${ }^{56}$ However, there is some evidence that IL-6 and PGE2 may induce tolerogenic DCs and may even promote programmed cell death protein ligand (PD-L)1 expression, which plays a major role in tumour escape from the immune system. ${ }^{57,58}$ Indeed, some studies have shown that differentiation of bone marrow-derived DCs with GM-CSF and IL-15/IL-14 or FMS-like tyrosine kinase 3 ligands (FLT3L), as well as suppression of the PGE2 pathway, can produce more mature and immunogenic DC phenotypes than standard DC preparations. ${ }^{59-62}$ While differentiation with GM-CSF/IL-4 has been shown to preferentially expand the conventional DC type 2 subset and produce more allogeneic DCs and inflammatory mediators than FLT3L, the latter expands both conventional DC type 1 and 2 subsets and results in more efficient migration of DCs to lymph nodes with a separate cytokine profile. ${ }^{60-62}$ Overall, GM-CSF/IL-4 DC resemble inflammationemergent DCs whereas FT3L DCs represent steady-state resident DCs. ${ }^{61}$ Other cytokine cocktails including toll-like receptor ligands to induce immunostimulatory cytokines are also under evaluation. ${ }^{63}$

Various protocols for differentiation and maturation have been developed over the years. Early generation of mature DCs from human monocytes in vitro required differentiation with GM-CSF and IL-4 for 5-7 days, followed by 2-3 days of activation. In 2003, a protocol was developed for differentiation and maturation of monocyte-derived DCs within 48 hours using in vitro culture, which was termed FastDC. Not only does a shorter protocol save labour, cost and time, but it also more closely models the differentiation of DCs in vivo. ${ }^{64}$ Monocytes were incubated for 24 hours with GM-CSF and IL-4, followed by activation with proinflammatory mediators for a further $24 \mathrm{~h} .64,65$ This protocol was later adapted for large-scale clinical use, which involved collection of MNCs by apheresis, isolation of monocytes by the Elutra Cell Separation System, and culturing in sterile Teflon bags. ${ }^{66}$ This manufacturing method has now been transferred into a closed-system (to minimise the risk of contamination), validated, GMP-compatible protocol, which may pave the way for larger multi-centre clinical trials. ${ }^{67}$ Overall, with its shorter incubation times, lower risk of contamination and large-scale production, FastDC is perhaps the most appropriate protocol in most situations; as a result it is widely used in clinical practice.

\section{Antigen loading of dendritic cells}

Following collection and enrichment, DCs must be loaded with antigens. Tumour antigens are frequently inconsistent in their expression between and within tumours, therefore loading DCs with peptides derived from multiple antigenic proteins improves the chance of recognition.53.68 Human DCs may be loaded with whole tumour-associated antigens; 
the most widely used method is incubation with human leukocyte antigen (HLA) class I- and class II-binding peptide. However, one potential problem with protein loading is the limited half-life of antigen expression on the DC surface..$^{53}$ An increasingly used alternative approach is to transfect DCs with tumour RNA-encoding antigens that, via continuous gene expression, can maintain antigen expression for the life of the DC. ${ }^{53}$ Examples include AGS-003, which has high transfection efficiencies and is well tolerated with only mild injection site reactions. ${ }^{9}$ When specific tumour antigens are unknown, other DC loading strategies may be used; these include pulsing with the beta-galactosidase protein, $\mathrm{H}-2 \mathrm{~K}$ restricted peptide, tumour cell lysates (prepared using freeze-thaw cycles; promising results have also been observed with squaric acid treatments of cell lysates ${ }^{69}$ ), and irradiated tumour cells and electrofusion of DCs with tumour cells. ${ }^{70}$ Of these, there is evidence that DC-tumour fusion cells may provide the most effective vaccines to treat existing tumours. ${ }^{9}$

More recently, it has been shown in a preclinical study have shown that novel antigen-capturing nanoparticles can deliver tumour-specific proteins to APCs, improving the efficacy of anti-PD-1 therapy in murine models of melanoma. ${ }^{11}$ This potentially offers a new strategy for the synergistic improvement of cancer immunotherapies that could be applied to the antigen loading of DC vaccines. ${ }^{71}$ In situ vaccines are also in development, which target DCs within the tumour microenvironment through intratumoural injections of toll-like receptor ligands or FLT3L, resulting in recruitment and local activation of DCs in the tumour. ${ }^{72}$ Overall, the choice of procedure depends on both the knowledge of antigens expressed by the tumour, and the availability of tumour tissues or other clinical grade sources of antigen. ${ }^{53}$

\section{Administration of dendritic cell-based vaccines}

Administration of DC vaccines is simple and the clinical resources required are minimal; a typical procedure involves thawing the cells for administration and subsequent injection of the cells. However, the success of DC-based vaccines depends on accurate delivery of cells. Delivery is typically intravenous, ${ }^{73,74}$ but subcutaneous, ${ }^{73}$ intradermal, ${ }^{74,75}$ intranasal, ${ }^{74}$ intraperitoneal, ${ }^{7,76}$ intratumoral ${ }^{77}$ and intranodal ${ }^{75}$ injection modes have been evaluated. ${ }^{73-75}$ Effective migration of DCs to the lymph nodes is essential in advanced cancer to interact with naive T cells, but DCs tend not to migrate easily. Intravenous administration may be quick and simple; however it may allow preferential retention of some cellular therapeutic agents in the liver. ${ }^{78}$

Similarly, subcutaneous or intradermal routes may allow only a modest fraction of DCs to enter the lymph nodes, and the long migratory times may reduce the ability of DCs to produce the required cytokines and chemokines. ${ }^{78}$ To overcome this, intranodal delivery has been attempted in melanoma patients with mixed success, but this route can be technically challenging and may lead to DC accumulation in perinodal

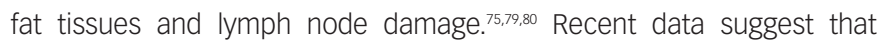
the prolonged (up to 7.5 weeks) delivery of DCs through implantable Iymphatic delivery ports is feasible and tolerable, though the potential advantages of this route of administration are yet to be confirmed. ${ }^{78}$

It is clear that further research is required to optimise the route of administration for DCs in specific indications and/or aid migration of DCs to the lymph nodes. Indeed, various methods are currently available to stimulate DC migration to the lymph nodes, including conditioning tissues using inflammatory cytokines, ${ }^{81}$ prostaglandins, ${ }^{82}$ chemokines, ${ }^{83}$ and matrix metalloproteinases. ${ }^{84}$ In vivo migration of magnetically labelled DCs can be tracked using magnetic resonance imaging to locate the cells in vivo. When used in vivo, this method can detect very low numbers of DCs, is safe and its use is feasible in humans. ${ }^{80}$ As the routine imaging protocols involved are readily available on conventional magnetic resonance imaging systems, it is hoped that this process may enable investigators and clinicians to obtain a deeper understanding of cellular treatment modalities and determine their respective efficacy. ${ }^{80}$

\section{Quality control measures for dendritic cells}

The clinical utility of DCs is dependent on effective means of quality control assessment. Therefore, there is a need for rapid methods to assess the product consistency related to the manufacturing process, as well as the identification of reliable quality assurance markers. The expression of classical DC markers, such as CD80, CD86, CD83 and positivity to $C C R 7,{ }^{85}$ can be measured using flow cytometry, but this is of limited use since these markers are expressed by DCs with different phenotypes. Gene expression profiling can be used to test the degrees of product consistency related to the manufacturing process and variability due to intra- and inter-donor factors, and how each factor affects single gene variation. For each gene, an index of variation is calculated and used to select candidate markers for identity testing, and also to define a set of genes that may be useful comparability and potency markers. This technique has proven effective, and has identified 29 potential markers for characterising DCs. ${ }^{85}$

Potency testing is useful to determine the functional capacity of the DC product, and the FDA recommends the development of potency assays for characterisation of cellular products used for human therapy. An IL12 p70 production assay has been developed that is applicable to small or large samples of DC vaccines generated under different conditions. The assay measures the ability to secrete IL-12p70 and respond to helper $T$ cell signals (CD4OL). It then quantifies IL-12p70 using an immunobead multiplex platform. The assay is reproducible, robust, and cost-effective, and can discriminate between DCs matured in the presence of different cytokine cocktails and between DCs obtained from healthy donors and patients with human immunodeficiency virus-1 or cancer. It is being used in ongoing early-phase clinical trials to determine its utility in predicting the in vivo efficacy of DC-based vaccines. ${ }^{86}$ Another novel method, the COSTIM bioassay, selectively measures co-stimulatory activity, or functional potency of the DCs. T cells are stimulated with a sub-optimal amount of anti-CD3 antibody and are unable to proliferate unless a source of co-stimulation (DCs) is added to the culture. ${ }^{87}$ In addition, the capacity to induce alloresponse (mixed lymphocyte reaction) is currently used to determine the potency of DC vaccine.

\section{Clinical application of dendritic cell-based vaccines}

Numerous DC-based anticancer therapeutics are in clinical development. ${ }^{88}$ Purified populations of CD1C (BDCA-1+) myeloid DCs were manufactured by pulsing autologous $C D 1 C^{+}$DCs, prepared by magnetic bead immunoselection from apheresed peripheral blood MNCs, with a cocktail of HLA-A*0201-restricted peptides and keyhole limpet haemocyanin, a potent immune-stimulating glycoprotein. The resulting vaccine was well tolerated in a phase I feasibility study in 12 patients with advanced metastatic prostate cancer, with preliminary data showing a mean survival of 18 months for $11 / 12$ patients and one patient remaining alive after over 5 years. ${ }^{39}$ In addition, some evidence of the induction of immune responses to control antigens was also observed (antikeyhole limpet hemocyanin antibodies in $25 \%$ of patients, delayed-type hypersensitivity to influenza matrix protein in $25 \%$ of patients). ${ }^{89}$ 
In patients with melanoma, Ridolfi et al. have reported a beneficial effect on survival using DCs prepared from peripheral blood MNCs using leukapheresis, adherence purification and pulsing with autologous tumour lysate; the median overall survival was 16 months, increasing to 22.9 months in patients who showed positive results in delayed-type hypersensitivity testing. ${ }^{21}$ Thus, there is a need for treatments aimed at improving immunological responsiveness to the vaccine, such as preleukapheresis radiotherapy and/or the addition of interferon alpha to the vaccine with the aim of increasing the vaccine-induced tumour immune response in patients with metastatic melanoma. ${ }^{90}$

More recently, naturally circulating DCs were used to vaccinate advanced

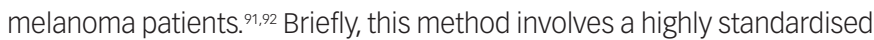
rapid isolation procedure with antibody-coated magnetic beads that produces clinically applicable purified DCs without an extensive culture period, which may have a positive effect on the immunological capacity. ${ }^{91,92}$ Once isolated from the patient and cultured overnight, the purified DCs are then activated, loaded with tumour-associated peptides and returned to the patient via intranodal injection. Results of two small studies ( $n=15$ and $n=14$ ) in patients with advanced melanoma have shown this approach to be feasible, with the induction of favourable immune responses (33-47\% showing tumour-antigen-specific CD8 ${ }^{+}$ T- cells in blood), minimal toxicity, evidence of improved progressionfree survival versus controls (4.0 versus 2.1 months) and even objective tumour responses in two patients. ${ }^{91,92}$ As with the previous melanoma studies, future phase III trials are needed to investigate the potential survival benefit.

\section{Future perspectives}

DC vaccines present a variety of opportunities for combination therapy with other complementary immunological cancer treatments to enhance the initial anti-tumour effect, as well as protecting against future remissions. This is because of their central co-ordinating role in both the innate and adaptive immune systems; in particular, their use in combination with checkpoint inhibitors could synergise both immune responses and the inhibitory pathways developed by cancer cells to evade immune responses. ${ }^{2}$ Other combined treatment approaches with DC vaccines include modulation of angiogenic pathways, depletion of soluble inhibitors, and adoptive transfer of T cells, which may be genetically modified to resist $T$ cell-directed tumour-immune evasion strategies. ${ }^{53}$

The use of DCs in cancer therapy offers the potential of personalised medicine; a DC vaccine loaded with tumour-specific amino acid substitutions (neoantigens) is being investigated in patients with advanced melanoma. ${ }^{93}$ However, a major disadvantage of this form of immunotherapy is the requirement for vaccines that are tailor-made for each individual (patient-specific). This is a substantial technical challenge involving large scale production and extensive resource utilisation to prepare every individual course of treatment.
Universal implementation of the technique will only be possible if more cost-effective and efficient strategies are developed. These challenges may be resolved with novel methods that are under investigation, which will target antigens to distinct DC subsets and simultaneously employ adjuvants to activate these cells to induce immunity. ${ }^{94,95}$ These subsets include monocyte-derived DCs, conventional DC type 1, conventional DC type 2, and plasmacytoid DCs. ${ }^{96}$ Monocyte-derived DCs are most frequently used for vaccination purposes, based on technical aspects such as their availability and in vitro expansion. ${ }^{96}$ However, recent technological developments have enabled the efficient isolation of conventional and plasmacytoid DCs directly from peripheral blood. ${ }^{96}$

Naturally occurring DCs have shown promising results, and have indicated different efficacy with different DC subsets. ${ }^{96}$ For example, identifying whether the immunosuppressive environment of the tumour consists of regulatory T cells (Tregs) or tumour-associated macrophages may help in selecting the optimal DC subset (in this case conventional DC type 1 or 2 , respectively) to induce the appropriate T-cell skewing. ${ }^{96}$ Similarly, modelling studies assessing the immune interactions of neo antigens in specific tumours have shown that neoantigens may act as a predictor of response to immunotherapy. ${ }^{97}$ As such, neoantigens may be useful in developing novel immunotherapies and personalised neoantigen-targeting vaccines. ${ }^{97,98}$

\section{Summary and concluding remarks}

The manufacture of DC vaccines is a complex process involving a number of technical challenges, including achieving the required yield for clinical use with appropriate purity, avoiding contamination by other blood components (e.g. platelets and RBCs), efficient maturation and antigen loading to produce viable mature DCs, and optimal administration of the vaccine to induce immunological responses in most patents. In addition, variation of MNC recruitment in donors has also been reported, though this requires further investigation. ${ }^{99}$

In recent years, the methods of manufacture have evolved and have started to meet these challenges, as well as improving convenience for the donor by reducing procedure time and product volume. ${ }^{45}$ The approaches for antigen loading have broadened, novel maturation signals have been developed and different sites of administration have been evaluated. As a result, robust, validated and GMP-compliant protocols have been developed for DC production.

Further development of these protocols is needed to produce standardised procedures for the preparation of cancer vaccines. Additionally, further refinements in vaccine strategies are needed to develop this promising therapeutic modality into a clinically meaningful treatment. This will facilitate larger multicentre clinical trials, with the aim of achieving widespread clinical adoption of therapeutic DC-based vaccines and potentially improved outcomes in a range of different cancer types. \
1. Guo C, Manjili MH, Subjeck JR, et al. Therapeutic cancer vaccines: past, present, and future. Adv Cancer Res. 2013:119:421-75. Palucka K, Banchereau J. Cancer immunotherapy via dendritic cells. Nat Rev Cancer. 2012;12:265-77.

Banchereau J, Steinman RM. Dendritic cells and the control of immunity. Nature. 1998:392:245-52.

4. Eyrich M, Rachor J, Schreiber SC, et al. Dendritic cell vaccination in pediatric gliomas: lessons learnt and future perspectives. Front Pediatr. 2013:1:12

5. Caruso DA, Orme LM, Amor GM, et al. Results of a Phase study utilizing monocyte-derived dendritic cells pulsed with tumor RNA in children with Stage 4 neuroblastom Cancer. 2005;103:1280-91.

6. Engell-Noerregaard L, Hansen $\mathrm{TH}$, Andersen $\mathrm{MH}$, et al. Review of clinical studies on dendritic cell-based vaccination of patients with malignant melanoma: assessment of correlation between clinical response and vaccine parameters. Cancer Immunol Immunother. 2009;58:1-14.

7. Chu CS, Boyer J, Schullery DS, et al. Phase I/ll randomized trial of dendritic cell vaccination with or without cyclophosphamide for consolidation therapy of advanced ovarian cancer in first or second remission cancer Immunol Immunother. 2012:61:629-41.

8. Wang $X$, Zhao HY, Zhang FC, et al. Dendritic cell-based vaccine for the treatment of malignant glioma: a systematic review. Cancer Invest. 2014:32:451-7.

9. Amin A, Dudek AZ, Logan TF, et al. Survival with AGS-003, an autologous dendritic cell-based immunotherapy, in combination with sunitinib in unfavorable risk patients with advanced renal cell carcinoma (RCC): Phase 2 study results I Immunother Cancer. 2015;3:14.

10. Pyzer AR, Avigan DE, Rosenblatt J. Clinical trials of dendritic cell-based cancer vaccines in hematologic malignancies. Hum Vaccin Immunother. 2014;10:3125-31.

11. Akiyama Y, Oshita C, Kume A, et al. Alpha-type-1 polarized dendritic cell-based vaccination in recurrent highgrade glioma: a phase I clinical trial BMC Cancer. 2012:12:623.

2. Oshita $C$ Takikawa M Kume A et a Dendritic cell-based oshin C, Tak in metastatic me, et al. Denditic cel-based vacination in metastic melanoma

. Nair S, Archer GE, Tedder TF. Isolation and generation of human dendritic cells. Curr Protoc Immunol. 2012; Chapter 7:Unit7 32.

14. Syme R, Bajwa R, Robertson L, et al. Comparison of CD34 and monocyte-derived dendritic cells from mobilized peripheral blood from cancer patients. Stem Cells. 2005;23:74-81

15. Anguille $S$, Smits EL, Bryant C, et al. Dendritic cells as pharmacological tools for cancer immunotherapy. 
Pharmacol Rev. 2015;67:731-53.

16. Fong $L$, Hou Y, Rivas A, et al. Altered peptide ligand vaccination with Flt3 ligand expanded dendritic cells for tumor immunotherapy. Proc Natl Acad Sci U S A. 2001:98:8809-14.

17. Hsu FJ, Benike C, Fagnoni $F$, et al. Vaccination of patients with B-cell lymphoma using autologous antigen-pulsed dendritic cells. Nat Med. 1996;2:52-8.

18. Wu K, Meng JS, Baihetiya A, et al. [Clinical efficacy and relative factors of dendritic cell-based tumor vaccination for prostate cancer: a systematic review and meta-analysis]. Article in Chinese. Zhonghua Nan Ke Xue. 2013;19:545-50.

19. Caballero-Banos M, Benitez-Ribas D, Tabera J, et al. Phase II randomised trial of autologous tumour lysate dendritic cell plus best supportive care compared with best supportive care in pre-treated advanced colorectal cancer patients. Eur I Cancer. 2016;64:167-74

20. Aarntzen EH, Bol K, Schreibelt G, et al. Skin-test infiltrating lymphocytes early predict clinical outcome of dendritic cell-based vaccination in metastatic melanoma. Cancer Res. 2012;72:6102-10.

21. Ridolfi L Petrini M, Fiammenghi L, et al. Dendritic cell-based vaccine in advanced melanoma: update of clinical outcome. Melanoma Res. 2011;21:524-9.

22. Vilella R, Benitez D, Mila J, et al. Pilot study of treatment of biochemotherapy-refractory stage IV melanoma patients with autologous dendritic cells pulsed with a heterologous melanoma cell line lysate. Cancer Immunol Immunother. 2004;53:651-8.

23. Aarntzen EH, Schreibelt G, Bol K, et al. Vaccination with mRNA-electroporated dendritic cells induces robust tumor antigen-specific CD4+ and CD8+ T cells responses in stage III and IV melanoma patients. Clin Cancer Res. 2012;18:5460-70.

24. Goyne HE, Cannon MJ. Dendritic cell vaccination, immune regulation, and clinical outcomes in ovarian cancer. Front Immunol. 2013:4:382.

25. Daga A, Bottino C, Castriconi R, et al. New perspectives in glioma immunotherapy. Curr Pharm Des. 2011;17:2439-67.

26. Johnson LA, Sampson JH. Immunotherapy approaches for malignant glioma from 2007 to 2009. Curr Neurol Neurosci Rep 2010;10:259-66

27. Lasky JL 3rd, Panosyan EH, Plant A, et al. Autologous tumor lysate-pulsed dendritic cell immunotherapy for pediatric patients with newly diagnosed or recurrent high-grade gliomas. Anticancer Res. 2013;33:2047-56.

28. Okamoto M, Kobayashi M, Yonemitsu Y, et al. Dendritic cell-based vaccine for pancreatic cancer in Japan. World I Gastrointest Pharmacol Ther. 2016;7:133-8.

29. Kantoff PW, Higano CS, Shore ND, et al. Sipuleucel-T immunotherapy for castration-resistant prostate cance N Engl J Med. 2010;363:411-22.

30. Cheever MA, Higano CS. PROVENGE (Sipuleucel-T) in prostate cancer: the first FDA-approved therapeutic cancer vaccine. Clin Cancer Res. 2011;17:3520-6.

31. Datta J, Terhune JH, Lowenfeld L, et al. Optimizing dendritic cell-based approaches for cancer immunotherapy Yale I Biol Med. 2014;87:491-518.

32. MacDonald KP, Munster DJ, Clark GJ, et al. Characterization of human blood dendritic cell subsets. Blood. 2002;100:4512-20.

33. Banchereau J, Palucka AK, Dhodapkar M, et al. Immune and clinical responses in patients with metastatic melanoma to CD34(+) progenitor-derived dendritic cell vaccine. Cancer Res. 2001;61:6451-8

34. Maraskovsky E, Brasel $\mathrm{K}$, Teepe $\mathrm{M}$, et al. Dramatic increase in the numbers of functionally mature dendritic cells in Flt3 ligand-treated mice: multiple dendritic cell subpopulations identified. J Exp Med. 1996;184:1953-62.

35. Romani N, Gruner S, Brang D, et al. Proliferating dendritic cell progenitors in human blood. J Exp Med. 1994;180:83-93.

6. Tazbirkova A, Okai M, Horley DC, et al. Effects of leukapheresis protocol, cell processing and cryopreservation on the generation of monocyte-derived DC for immune therapy. Cytotherapy. 2003;5:31-9.

37. Liau LM, Prins RM, Kiertscher SM, et al. Dendritic cell vaccination in glioblastoma patients induces systemic and intracranial T-cell responses modulated by the local centra nervous system tumor microenvironment Clin Cancer Res. 2005;11:5515-25.

38. Strasser EF, Berger TG, Weisbach V, et al. Comparison of two apheresis systems for the collection of CD14+ cells intended to be used in dendritic cell culture. Transfusion. 2003;43:1309-16.

39. Strasser EF, Dittrich S, Weisbach V, et al. Comparison of two mononuclear cell program settings on two apheresis devices intended to collect high yields of CD14+ and CD3+ cells. Transfusion. 2004;44:1104-11.

40. Wolf CE, Meyer M, Riggert J. Leukapheresis for the extraction of monocytes and various lymphocyte subpopulations from peripheral blood: product quality and prediction of the yield using different harvest procedures. Vox Sang. 2005;88:249-55.

41. Strasser EF, Hendelmeier M, Weisbach V, et al. CD 14+ cell collection in non-cytokine-stimulated donors with the COM.TEC cell separator. Transfusion. 2006;46:66-73.

42. Steininger PA, Smith R, Geier C, et al. Leukapheresis in noncytokine-stimulated donors with a new apheresis system: first-time collection results and evaluation of subsequent cryopreservation. Transfusion. 2013;53:747-56.

43. Andreassen GS, Skoge L, Lundby M, et al. Spectra Optia and Andreassen GS, Skoge L, Lundby M, et al. Spectra Optia and
Elutra for the production of monocyte-derived dendritic cell Elutra for the production of monocyte-derived
vaccines. Cytotherapy. 2014:16(suppl):S46.

44. Steininger PA, Strasser EF, Weiss D, et al. First comparative evaluation of a new leukapheresis technology in non-cytokinestimulated donors. Vox Sang. 2014;106:248-55.

45. Punzel M, Kozlova A, Quade A, et al. Evolution of MNC and Iymphocyte collection settings employing different Spectra Optia(R) Leukapheresis systems. Vox Sang 2017:112:586-94.

46. Lee SN, Sohn JY, Kong JH, et al. Comparison of Two Apheresis systems of COBE and Optia for Autologous Peripheral Blood Stem Cell Collection. Ann Lab Med. 2017;37:327-30.

47. Weyrich AS, Elstad MR, MCEver RP, et al. Activated platelets signal chemokine synthesis by human monocytes. I Clin Invest. 1996;97:1525-34

48. Burk S, Erdmann M, Weiss D, et al. Buffy coat volume reduction for optimization of leucapheresis harvests produced by the autoMNC program. Vox Sang. 2015;108:89-95.

49. Felzmann T, Witt V, Wimmer D, et al. Monocyte enrichment from leukapharesis products for the generation of DCs by plastic adherence, or by positive or negative selection. Cytotherapy. 2003:5:391-8.

50. Wong EC, Lee SM, Hines K, et al. Development of a closedsystem process for clinical-scale generation of DCs: evaluation of two monocyte-enrichment methods and two culture containers. Cytotherapy 2002:4:65-76.

51. ten Brinke A, Karsten ML, Dieker MC, et al. Generation of dendritic cells for immunotherapy is minimally impaired by granulocytes in the monocyte preparation. Immunobiology. 2006;211:633-40.

52. Kim S, Kim HO, Baek EJ, et al. Monocyte enrichment from eukapheresis products by using the Elutra cell separator. Transfusion. 2007;47:2290-6.

5. Turnis $\mathrm{ME}$, Rooney $\mathrm{CM}$. Enhancement of dendritic cells as vaccines for cancer. Immunotherapy. 2010;2:847-62.

54. De Vries IJ, Krooshoop DJ, Scharenborg NM, et al. Effective migration of antigen-pulsed dendritic cells to lymph nodes in melanoma patients is determined by their maturation state. Cancer Res. 2003;63:12-7.

55. de Vries 4 Lesterhuis WI, Scharenborg NM, et al. Maturation of dendritic cells is a prerequisite for inducing immune responses in advanced melanoma patients. Clin Cancer Res. 2003;9:5091-100

56. Jonuleit H, Kuhn U, Muller G, et al. Pro-inflammator cytokines and prostaglandins induce maturation of potent mmunostimulatory dendritic cells under fetal calf serum-free conditions. Eur I Immunol. 1997;27:3135-42.

57. Prima V, Kaliberova LN, Kaliberov S, et al. COX2/mPGES1/PGE2 pathway regulates PD-L1 expression in tumor-associated macrophages and myeloid-derived suppressor cells. Proc Nat Acad Sci U S A. 2017;114:1117-22.

58. Torres-Aguilar H, Sanchez-Torres C, Jara L, et al. IL-10/TGF-betatreated dendritic cells, pulsed with insulin, specifically reduce the response to insulin of $\mathrm{CD} 4+$ effector/memory $\mathrm{T}$ cells from the res

59. Mookerjee A, Graciotti M, Kandalaft LE. IL-15 and a two-step maturation process improve bone marrow-derived dendritic cell cancer vaccine. Cancers (Basel). 2019;11:40

60. Parajuli P, Mosley RL, Pisarev V, et al. Flt3 ligand and granulocyte-macrophage colony-stimulating facto preferentially expand and stimulate different dendritic and T-cell subsets. Exp Hematol. 2001;29:1185-93.

61. Xu Y, Zhan Y, Lew AM, et al. Differential development of murine dendritic cells by GM-CSF versus Flt3 ligand has implications for inflammation and trafficking. J Immunol. 2007:179:7577-84.

62. Weigel BJ, Nath N, Taylor PA, et al. Comparative analysis of murine marrow-derived dendritic cells generated by Flt3L or GM-CSF/IL-4 and matured with immune stimulatory agents on the in vivo induction of antileukemia responses. Blood. 2002:100:4169-76.

63. Massa C, Thomas C, Wang E, et al. Different maturation cocktails provide dendritic cells with different chemoattractive properties. J Trans/ Med. 2015;13:175.

64. Dauer M, Obermaier B, Herten J, et al. Mature dendritic cells derived from human monocytes within 48 hours: a novel strategy for dendritic cell differentiation from blood precursors. I Immunol. 2003;170:4069-76.

65. Obermaier B, Dauer M, Herten J, et al. Development of a new protocol for 2-day generation of mature dendritic cells from human monocytes. Biol Proced Online. 2003;5:197-203.

66. Jarnjak-Jankovic $\mathrm{S}$, Hammerstad $\mathrm{H}$, Saeboe-Larssen $\mathrm{S}$, et al. A full scale comparative study of methods for generation of functional Dendritic cells for use as cancer vaccines $B M C$ Cancer. 2007;7:119.

67. Eyrich M, Schreiber SC, Rachor J, et al. Development and validation of a fully GMP-compliant production process of autologous, tumor-lysate-pulsed dendritic cells. Cytotherapy. 2014:16:946-64

68. Fay JW, Palucka AK, Paczesny S, et al. Long-term outcomes in patients with metastatic melanoma vaccinated with melanom peptide-pulsed CD34(+) progenitor-derived dendritic cells. Cancer Immunol Immunother. 2006;55:1209-18.

69. Mookerjee A, Graciotti M, Kandalaft L. A cancer vaccine with dendritic cells differentiated with GM-CSF and IFNalpha and pulsed with a squaric acid treated cell lysate improves T cell priming and tumor growth control in a mouse model. Bioimpacts. 2018:8:211-21.

70. Shimizu K, Kuriyama H, Kjaergaard J, et al. Comparative analysis of antigen loading strategies of dendritic cells for tumor immunotherapy. I Immunother 2004:27:265-7 2 .

71. Min Y, Roche KC, Tian S, et al., Antigen-capturing nanoparticles improve the abscopal effect and cancer immunotherapy. Nat Nanotechnol. 2017:12:877-82.

72. Saxena M, Balan S, Roudko V et al Towards superior dendriticcell vaccines for cancer therapy. Nat Biomed Eng. 2018;2:341-6.

73. Curti A, Tosi P, Comoli P, et al. Phase I//l clinical trial of sequential subcutaneous and intravenous delivery of dendritic cell vaccination for refractory multiple myeloma using patientspecific tumour idiotype protein or idiotype (VDJ)-derived class I-restricted peptides. Br J Haematol. 2007:139:415-24.

74. Hangalapura BN, Oosterhoff D, Gupta T, et al. Delivery route MyD88 signaling and cross-priming events determine the anti-tumor efficacy of an adenovirus based melanoma vaccine Vaccine. 2011;29:2313-21.

75. Lesterhuis WJ, de Vries IJ, Schreibelt G, et al. Route of administration modulates the induction of dendritic cell vaccine-induced antigen-specific T cells in advanced melanoma patients. Clin Cancer Res. 2011;17:5725-35.

76. Jauregui-Amezaga A, Cabezon R, Ramirez-Morros A, et al. Intraperitoneal administration of autologous tolerogenic dendritic cells for refractory Crohn's disease: a phase I study. J Crohns Colitis. 2015;9:1071-8.

77. Raich-Regue D, Fabian KP, Watson AR, et al. Intratumoral delivery of mTORC2-deficient dendritic cells inhibits B16 melanoma growth by promoting CD8(+) effector T cell responses. Oncoimmunology. 2016;5:e1146841.

78. Radomski M, Zeh HJ, Edington HD, et al. Prolonged intralymphatic delivery of dendritic cells through implantable lymphatic ports in patients with advanced cancer. I Immunother Cancer. 2016;4:24

79. Verdijk P, Aarntzen EH, Lesterhuis WJ, et al. Limited amounts of dendritic cells migrate into the T-cell area of lymph nodes but have high immune activating potential in melanoma patients. Clin Cancer Res. 2009;15:2531-40.

80. de Vries IJ, Lesterhuis WJ, BarentsZ JO, et al. Magnetic resonance tracking of dendritic cells in melanoma patients for monitoring of cellular therapy. Nat Biotechnol. 2005;23:1407-13.

81. Martin-Fontecha A, Sebastiani S, Hopken UE, et al. Regulation of dendritic cell migration to the draining lymph node: impact on T lymphocyte traffic and priming. J Exp Med. 2003;198:615-21.

82. Boullart AC, Aarntzen EH Verdik P, et al Maturation of monocyte-derived dendritic cells with Toll-like receptor 3 and $7 / 8$ ligands combined with prostaglandin E2 results in high interleukin-12 production and cell migration. Cancer Immuno Immunother. 2008;57:1589-97.

83. Vicari AP, Vanbervliet B, Massacrier C, et al. In vivo manipulation of dendritic cell migration and activation to elicit antitumour immunity. Novartis Found Symp. 2004;256:241-54; discussion 54-69.

84. Ratzinger G, Stoitzner P, Ebner S, et al. Matrix metalloproteinases 9 and 2 are necessary for the migration of Langerhans cells and dermal dendritic cells from human and murine skin. J Immunol. 2002;168:4361-71.

85. Castiello L, Sabatino M, Zhao Y, et al. Quality controls in cellular immunotherapies: rapid assessment of clinical grade dendritic cells by gene expression profiling. Mol Ther. 2013;21:476-84.

86. Butterfield $L H$, Gooding W, Whiteside TL, Development of a potency assay for human dendritic cells: IL-12p70 production. Immunother. 2008:31:89-100.

87. Shankar G, Fourrier MS, Grevenkamp MA, et al. Validation of the COSTIM bioassay for dendritic cell potency. J Pharm Biomed Anal. 2004;36:285-94

88. Garg AD, Vara Perez M, Schaaf M, et al. Trial watch: Dendritic cell-based anticancer immunotherapy. Oncoimmunology. 2017;6:e1328341.

89. Prue RL, Vari F, Radford KJ, et al. A phase I clinical trial of CD1C (BDCA-1)+ dendritic cells pulsed with HLA-A*0201 peptides for immunotherapy of metastatic hormone refractory prostate cancer. I Immunother. 2015;38:71-6.

90. de Rosa F, Ridolfi L. Ridolfi R, et al Vaccination with autologous dendritic cells loaded with autologous tumor lysate or homogenate combined with immunomodulating radiotherapy and/or preleukapheresis IFN-alpha in patients with metastatic melanoma: a randomised "proof-of-principle" phase II study. J Transl Med. 2014;12:209.

91. Tel J, Aarntzen EH, Baba T, et al. Natural human plasmacytoid dendritic cells induce antigen-specific T-cell responses in melanoma patients. Cancer Res. 2013;73:1063-75.

92. Schreibelt G, Bol KF, Westdorp H, et al. Effective clinical responses in metastatic melanoma patients after vaccination with primary myeloid dendritic cells. Clin Cancer Res. 2016;22:2155-66.

93. Carreno BM, Magrini V, Becker-Hapak M, et al. Cancer immunotherapy. A dendritic cell vaccine increases the breadth and diversity of melanoma neoantigen-specific T cells. Science. 2015:348:803-8.

94. Tacken PJ, Figdor CG. Targeted antigen delivery and activation of dendritic cells in vivo: steps towards cost effective vaccines Semin Immunol. 2011:23:12-20.

95. Lehmann CHK, Heger L, Heidkamp GF, et al. Direct delivery of antigens to dendritic cells via antibodies specific for endocytic receptors as a promising strategy for future therapies. Vaccines (Basel). 2016;4:8

96. Huber A, Dammeijer F, Aerts J, et al. Current state of dendritic cell-based immunotherapy: opportunities for in vitro antigen loading of different DC subsets? Front Immunol. 2018;9:2804. 